\title{
Using the Movember Foundation's GAP3 cohort to measure the effect of active surveillance on patient-reported urinary and sexual function - a retrospective study in low-risk prostate cancer patients
}

\author{
R. Trafford Crump ${ }^{1 \#} \wedge$, Sebastiaan Remmers ${ }^{2 \#} \wedge$, Mieke Van Hemelrijck $^{3} \wedge$, Jozien Helleman ${ }^{2}$, Daan Nieboer ${ }^{2} \wedge$, \\ Monique J. Roobol ${ }^{2} \wedge$, Lionne D. F. Venderbos ${ }^{2}$; Movember Foundation's Global Action Plan Prostate \\ Cancer Active Surveillance (GAP3) consortium* \\ ${ }^{1}$ Department of Surgery, University of Calgary, Calgary, Canada; ${ }^{2}$ Department of Urology, Erasmus University Medical Center, Rotterdam, The \\ Netherlands; 'King's College London, Faculty of Life Sciences and Medicine, Translational Oncology \& Urology Research (TOUR), London, UK \\ Contributions: (I) Conception and design: All authors; (II) Administrative support: None; (III) Provision of study material or patients: None; (IV) \\ Collection and assembly of data: None; (V) Data analysis and interpretation: S Remmers, D Nieboer; (VI) Manuscript writing: All authors; (VII) \\ Final approval of manuscript: All authors. \\ \#These authors contributed equally to this work. \\ Correspondence to: Lionne D. F. Venderbos, PhD. Department of Urology, Erasmus University Medical Center Rotterdam, Wytemaweg 80, 3015 CN \\ Rotterdam, The Netherlands. Email: 1.venderbos@erasmusmc.nl.
}

\begin{abstract}
Background: Active surveillance (AS) for low-risk prostate cancer (PCa) is intended to overcome potential side-effects of definitive treatment. Frequent prostate biopsies during AS may, however, impact erectile (EF) and urinary function (UF). The objective of this study was to test the influence of prostate biopsies on patient-reported EF and UF using multicenter data from the largest to-date AS-database.

Methods: In this retrospective study, data analyses were performed using the Movember GAP3 database (v3.2), containing data from 21,169 AS participants from 27 AS-cohorts worldwide. Participants were included in the study if they had at least one follow-up prostate biopsy and completed at least one patient reported outcome measure (PROM) related to EF [Sexual Health Inventory for Men (SHIM)/five item International Index of Erectile Function (IIEF-5)] or UF [International Prostate Symptom Score (IPSS)] during follow-up. The longitudinal effect of the number of biopsies on either SHIM/IIEF-5 or IPSS were analyzed using linear mixed models to adjust for clustering at patient-level. Analyses were stratified by center; covariates included age and Gleason Grade group at diagnosis, and time on AS.

Results: A total of 696 participants completed the SHIM/IIEF-5 3,175 times, with a median follow-up of 36 months [interquartile range (IQR) 20-55 months]. A total of 845 participants completed the IPSS 4,061 times, with a median follow-up of 35 months (IQR 19-56 months). The intraclass correlation (ICC) was 0.74 for the SHIM/IIEF-5 and 0.68 for the IPSS, indicating substantial differences between participants' PROMs. Limited heterogeneity between cohorts in the estimated effect of the number of biopsies on either PROM were observed. A significant association was observed between the number of biopsies and the SHIM/IIEF-5 score, but not for the IPSS score. Every biopsy was associated with a decrease in the SHIM/IIEF-5 score of an average 0.67 (95\% CI, $0.47-0.88)$ points.
\end{abstract}

Conclusions: Repeated prostate biopsy as part of an AS protocol for men with low-risk PCa does not

\footnotetext{
*The Movember Foundation's Global Action Plan Prostate Cancer Active Surveillance (GAP3) consortium members presented in Appendix 1.

^ ORCID: R. Trafford Crump, 0000-0001-6690-1926; Monique J. Roobol, 0000-0001-6967-1708; Sebastiaan Remmers, 0000-0001-95306448; Daan Nieboer, 0000-0002-4403-6537; Mieke Van Hemelrijck; 0000-0002-7317-0858.
} 
have a significant association with self-reported UF but does impact self-reported sexual function. Further research is, however, needed to understand whether the effect on sexual function implies a negative clinical impact on their quality of life and is meaningful from a patient's perspective. In the meantime, clinicians and patients should anticipate a potential decline in erectile function and hence consider incorporating the risk of this harm into their discussion about opting for AS and also when deciding on the stringency of follow-up biopsy schedules with long-term AS.

Keywords: Prostate cancer (PCa); patient-reported outcome; Sexual Health Inventory for Men (SHIM); five item International Index of Erectile Function (IIEF-5); International Prostate Symptom Score (IPSS)

Submitted Sep 10, 2020. Accepted for publication Apr 29, 2021.

doi: $10.21037 /$ tau-20-1255

View this article at: http://dx.doi.org/10.21037/tau-20-1255

\section{Introduction}

Active surveillance (AS) is included in the national and international guidelines as a viable management strategy for men diagnosed with low-risk, localized prostate cancer ( $\mathrm{PCa})$ (1-3). AS involves regular follow-up testing through prostatespecific antigen (PSA) tests, digital rectal examination (DRE), repeat prostate biopsy, and, when indicated, the use of prostate imaging (4). The goal of AS is to monitor lowrisk tumors instead of directly treating them with definitive treatment such as radical prostatectomy or radiotherapy. In case of reclassification or progression of the disease, switching to definitive treatment is still possible. So far, low rates of metastases and disease-specific mortality have been reported for men following an AS trajectory long term, which has resulted in the increased use of AS internationally (5-8).

Although side-effects of definitive treatment may be overcome or postponed by choosing an initial AS monitoring strategy, there are risks of potential harms to the patient associated with AS (9). This risk stems largely from repeated biopsy of the prostate, which can cause local inflammation, urosepsis, trauma, and-for peripherally directed biopsies-put periprostatic vasculature and nerves at risk (10). This can have a direct impact on functional outcomes, notably urinary and sexual functions. To-date, the evidence with respect to urinary function (UF) suggests a minimal impact $(11,12)$; the evidence on sexual function, however, is more mixed (13-16). The generalizability of the predominantly single-center studies into the effect of repeated prostate biopsies on men's urinary and sexual outcomes while on AS for low-risk PCa is limited by their sample size, the number of care centers involved, and the AS protocols involved.

There is an opportunity to overcome these limitations through the Movember Foundation's Global Action Plan, Prostate Cancer Active Surveillance (GAP3), where multiple sites representing a variety of AS protocols have contributed to an international longitudinal database (4). This retrospective study's objective was to leverage the GAP3 database to assess the effect of repeated prostate biopsies on men's self-reported urinary and sexual functions. The results from this study could be used several ways. For clinicians, it could help inform their own AS protocols in weighing the risks of potential harms and benefits associated with serial biopsy of the prostate. For patients, these results could help inform their treatment decision in terms of the potential functional outcomes associated with those options, including AS. We present the following article in accordance with the STROBE reporting checklist (available at http://dx.doi.org/10.21037/tau-20-1255).

\section{Methods}

\section{Study population}

This study performed a retrospective analysis of data collected as part of GAP3, the full details of which have been published elsewhere (4). In brief, patients diagnosed with PCa were included in GAP3 if, at the time of diagnosis, they were characterized as having: clinical stage T1-T2, serum PSA $\leq 10 \mathrm{ng} / \mathrm{mL}$, a biopsy Gleason grade group of 1 or 2 , and a maximum of two tumor-positive biopsy core samples were detected (i.e., low-risk PCa). Follow-up protocols were heterogeneous, but in general they recommended: serial measurements of serum PSA levels every 3 to 6 months, a DRE every 6 to 12 months, and prostate biopsy to identify pathological progression every 1 to 3 years. Version 3.2 of the Movember GAP3 
database contained data from 21,169 AS participants from 27 worldwide AS-cohorts. All participating study centers have obtained local ethics approval for their individual AS studies and subsequent data transfer to GAP3, including secondary analyses studies, such as the one reported here.

To be included in this study, GAP3 participants had to have undergone at least one follow-up prostate biopsy. They also had to have completed at least one of the following patient reported outcome measures (PROMs): the International Prostate Symptom Score (IPSS), or the Sexual Health Inventory for Men (SHIM) [also referred to as the five item International Index of Erectile Function (IIEF-5)]. Based on the above selection criteria, only data from the University of Calgary, the University of California San Francisco (UCSF) and the Instituti Tumori in Milan were used since other centers did not comply with the inclusion criteria.

\section{PROMs}

\section{IPSS}

The IPSS is comprised of eight items (17). The first seven items measure the type and degree of lower urinary tract symptoms (LUTS) experienced over the past month, including: frequency, nocturia, weak urinary stream, hesitancy, intermittence, incomplete emptying, and urgency. Each of these items is responded to using a five-point Likert scale ranging from 0 (i.e., "Not at all") to 5 (i.e., "Almost Always") (note that for the nocturia item, the description changes from "None" to "5 times or more"). The scores for the seven items are aggregated into the "Total IPSS Score" that ranges from 0 to 35, with higher scores representing more severe symptom severity. Three thresholds for the Total IPSS Score have been validated to indicate the severity of LUTS; a score of: 1 to 7 indicates mild, 8 to 19 indicates moderate, and 20 to 35 indicates severe symptoms (17). A minimal clinically important difference (MCID) — that is, the minimum change in the Total IPSS Score needed to be considered clinically meaningful-has been defined as four or more points $(17,18)$. A validated translation of the IPSS was used by the study center in Milan (Italian) (19).

The eighth IPSS item measures the effect of urinary symptoms on the respondent's quality-of-life. It is used more as a qualitative measure of symptom severity and is not included in the Total IPSS Score. It is not used in this study's analysis.

\section{SHIM/IIEF-5}

The SHIM is comprised of five items related to symptoms of erectile dysfunction experienced over the past six months, including: erectile function, orgasmic function, sexual desire, intercourse satisfaction, and overall satisfaction. The SHIM was developed as an abridged version of the 15 -item IIEF and is also referred to as the IIEF-5. Four items are responded to using a six-point Likert scale ranging from 0 (i.e., "Do Not Attempt Intercourse"/"No Sexual Activity") to 5 (i.e., "Almost Always or Always"/"Not Difficult"). A single item is responded to using a five-point Likert scale ranging from 1 (i.e., "Very Low") to 5 (i.e., "Very High"). The scores from all items are aggregated into a total score ranging from 1 to 25 , with higher scores representing less symptom severity. Five thresholds for the total score have been validated to indicate the severity of erectile dysfunction; a score of: 1 to 7 indicates severe, 8 to 11 indicate moderate, 12 to 16 indicates mild to moderate, 17 to 21 indicates mild, and 22-25 indicates no symptoms (20).

\section{Statistical analysis}

Because not all GAP3 participants completed both the IPSS and SHIM/IIEF-5 they were treated as separate samples. For each, the median and interquartile range (IQR) of participants' characteristics at inclusion were reported, as well as the baseline PROM score. The longitudinal measures of either the total IPSS score or the SHIM/IIEF5 total score were analyzed using linear mixed models to adjust for clustering at participant level using a random intercept for the participant. Analyses were stratified by center, and the coefficient for the parameter number of biopsies was pooled for all centers. The fixed effect included the total number of biopsies up to a particular PROMmoment; covariates included the age at diagnosis, the time on AS, and the Gleason Grade group at diagnosis. The intercept-only model estimated the intraclass correlation (ICC). The ICC describes the proportion of the total variance in PROM that pertains to differences between men and ranges from 0 to 1 , whereby a lower number represents less variance between men.

All statistical analyses were performed using $\mathrm{R}$ version 3.6.0 (21), complemented by R-package metaphor (22). Missing covariates (i.e., age at diagnosis) were imputed using the Bayesian framework with R-package jointAI (23).

\section{Ethical statement}

The study was conducted in accordance with the Declaration of Helsinki (as revised in 2013). The study 
was approved by institutional/regional/national ethics/ committee/ethics board of all 27 centers participating in the Movember GAP3 project. Between 2014 and 2016, the global GAP3 database was created by combining patient data from established AS cohorts worldwide. Requirements for participation included, amongst others, ethical approval for sharing digital patient data in a centralized global database and active registry of active surveillance patients. To date, 27 centers from the USA, Canada, Australasia, the UK, and Europe fulfilled the requirements for participation and joined the initiative. Each institution has obtained institution ethical approval and signed a Movember end user license agreement, an access rights principles agreement, and the commonly agreed upon GAP3 analytical plan.

\section{Results}

\section{Urinary symptoms}

A total of 845 participants fulfilled the inclusion criteria and completed the IPSS 4,061 times. Median follow-up was 35 months (IQR $=19-56$ months). Median age at inclusion was 64 years (IQR =59-69 years) (Table 1). At diagnosis, participants reported a median IPSS score of $8(\mathrm{IQR}=4-13)$. Participants underwent a median of 3 biopsies (IQR $=2-4$ biopsies, max 13).

The ICC was 0.68 , indicating large differences between participants' IPSS score. The estimated coefficients of number of biopsies ranged from 0.06 to 0.34 with relatively wide confidence intervals (CI) (Figure 1). Overall, there was no statistically significant association between IPSS score and the number of biopsies across the cohorts (pooled coefficient 0.09 (95\% CI, -0.07 to 0.25). Despite different inclusion criteria and follow-up schedules, results were consistent across cohorts.

\section{Erectile functioning}

A total of 696 participants fulfilled the inclusion criteria and completed the SHIM/IIEF-5 3,175 times. Median followup was 36 months (IQR $=20-55$ months). Median age at inclusion was 64 years (IQR =59-68 years) (Table 1). At diagnosis, participants reported a median SHIM/IIEF-5 score of 20 (IQR $=11-24)$. Participants underwent a median of 3 biopsies (IQR $=2-5$ biopsies, $\max 13$ ).

The ICC was 0.74 , indicating large differences between participants' SHIM/IIEF-5 score. The estimated coefficients of number of biopsies ranged from -0.76 to
-0.65 , with relatively wide CI's (Figure 2). Overall, every additional biopsy reduced the SHIM/IIEF-5 score an average of 0.67 points ( $95 \%$ CI, $0.47-0.88$ ).

\section{Conclusions}

This study aimed to measure the effect of repeated prostate biopsies conducted as part of an AS monitoring strategy for low-risk PCa on men's self-reported urinary and sexual function. We did this using the largest centralized AS database to date, which included IPSS and SHIM/IIEF5 response data, collected from multiple care centers worldwide. We observed that the number of prostate biopsies was not significantly associated with a change in self-reported UF, as measured by the IPSS. There was, however, a significant association between the number of prostate biopsies and self-reported erectile functioning, as measured by the SHIM/IIEF- 5 .

To our knowledge, this is the first study analyzing the effect of repeated prostate biopsies on self-reported urinary and erectile function using multi-center data. Earlier studies investigating similar associations have done so using data from single center settings $(9,12-14,16)$. Glass et al. concluded that performing repeat prostate biopsies does not increase the risk of harm to UF for men on AS for PCa (10). Braun et al. reported a small decrease in erectile dysfunction in men who underwent repeated biopsies. Participants in their cohort had a median age of 64 years (IQR $=58-68$ years), were on AS for a median of 3.5 years (IQR $=2.3-5.0$ years) and underwent a median number of 5 biopsies (IQR $=3-6$ ). A decrease in erectile function of 1.0 point per year (95\% CI, 0.2-1.7) was observed during the first four years on AS, as measured on the IIEF-6 scale (scale 1 to 30, with lower scores representing worse outcomes) (15). Pearce et al., who investigated the changes in sexual function in men who had been on AS for 24 months using the EPIC, did not observe a significant relationship with the number of prostate biopsies nor the number of cores per biopsy (16).

The results from our sexual function analysis indicate that every additional prostate biopsy reduces the SHIM/ IIEF- 5 score by an average of 0.67 points. That change is statistically significant, but whether it is clinically significant-or, more specifically, whether it represents a change that is meaningful from the patient's perspectivehas yet to be determined. A MCID—that is, the minimum change in the score needed to be considered meaningful- 
Table 1 Patient characteristics of men who completed the IPSS and/or the SHIM/IIEF-5

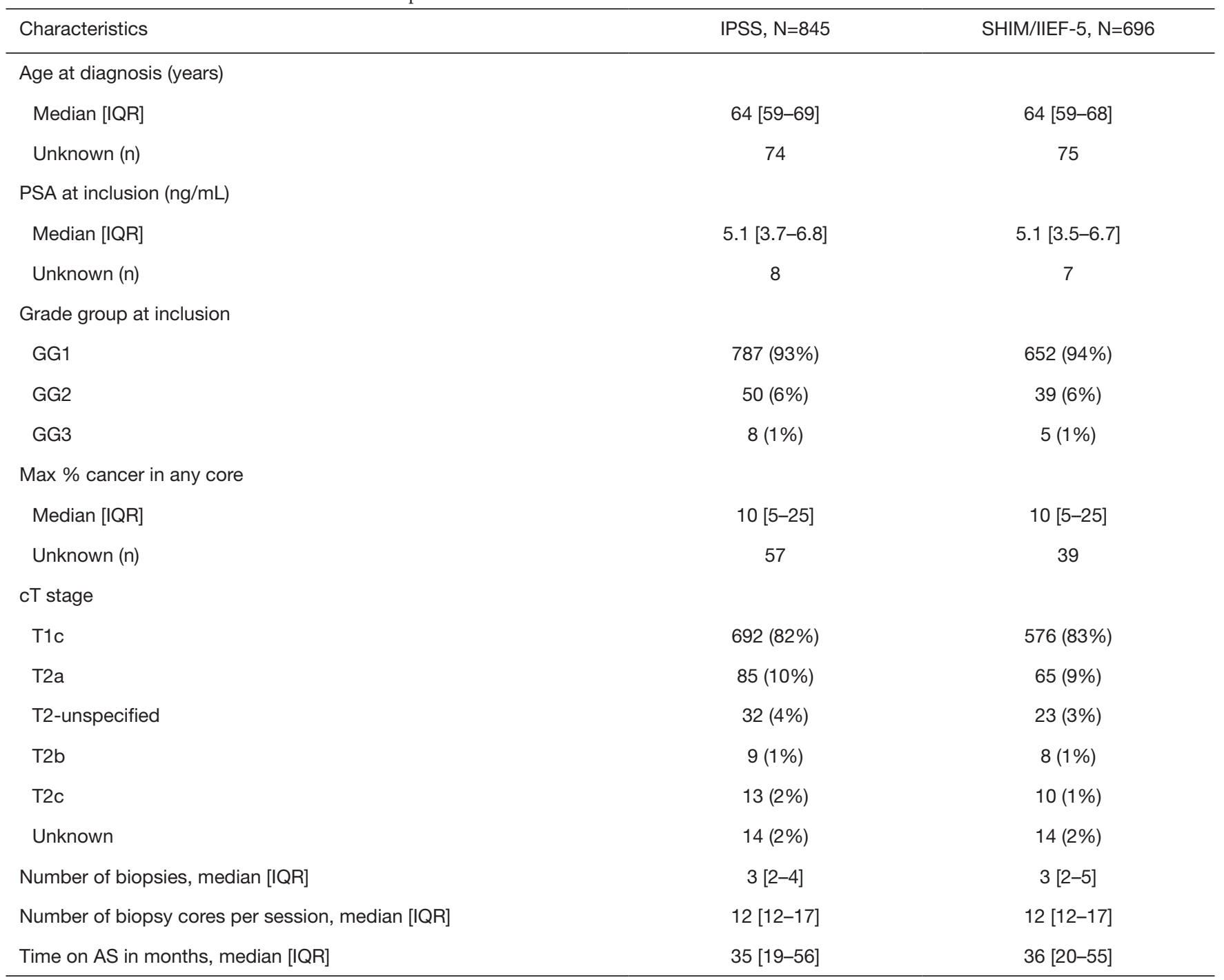

IPSS, International Prostate Symptom Score; SHIM, Sexual Health Inventory for Men; IIEF-5, five item International Index of Erectile Function; AS, active surveillance; IQR, interquartile range.

for the SHIM/IIEF-5 has not been established. It is possible to imagine that after a long period on AS the subsequent effect of undergoing multiple biopsy sessions, will have an impact. After for example, 5 biopsy sessions, the SHIM/ IIEF -5 score increases by $5 \times 0.67=3.35$ points. Whilst we note that erectile dysfunction increases with every additional prostate biopsy taken, we need to be aware that age has also been linked to lowered sexual function. We aimed to take into account the effects of age at baseline and time on AS, but there may always be some residual confounding.

Clinical guidelines for the treatment of $\mathrm{PCa}$ from various national and international associations, such as the American
Urology Association and the European Association of Urology, strongly recommend shared decision making when deciding on the appropriate management strategy for men with low-risk, localized $\mathrm{PCa}$ as the treatment decision is a preference sensitive one $(2,24)$. In shared decision making, the role of the clinician is to offer treatment options and describe their associated risks of harms and benefits upon which patients can arrive at more informed treatment preferences (25). Clinicians should consider incorporating the results of this study into their description of AS for low-risk PCa. While definitive treatments such as radical prostatectomy and radiotherapy come with traditionally 


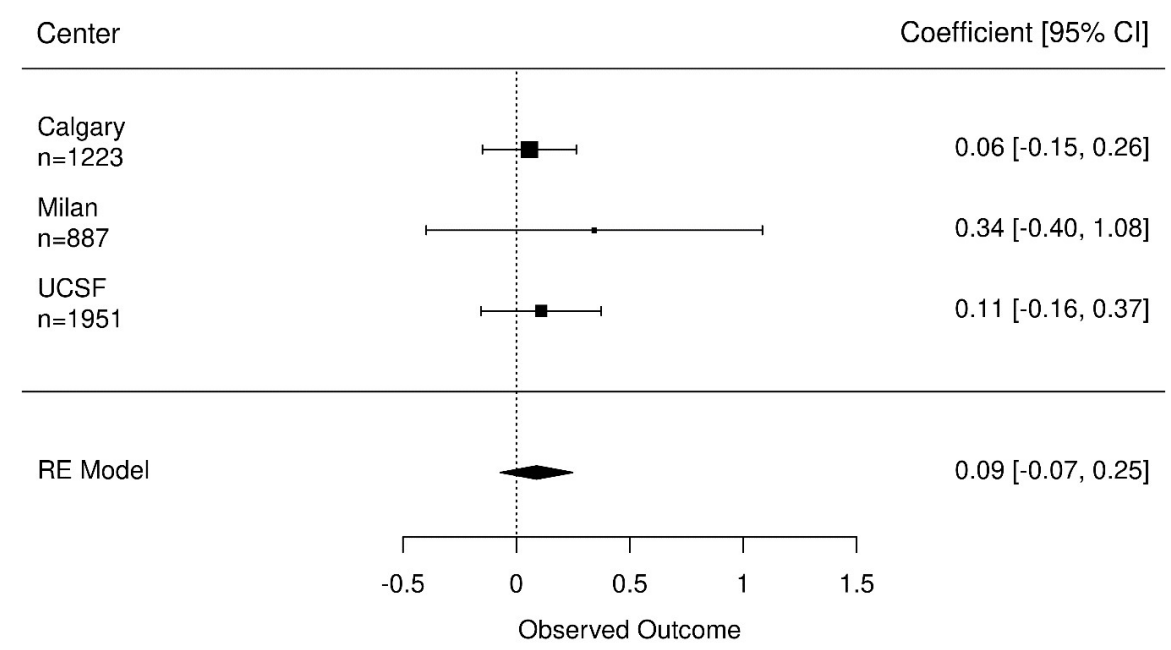

Figure 1 Coefficients of the number of biopsies on urinary functioning measured by the IPSS, n represent number of questionnaires completed. The effect of the number of biopsies on urinary functioning is controlled for age at diagnosis, time on AS and the Gleason score at diagnosis. AS, active surveillance.

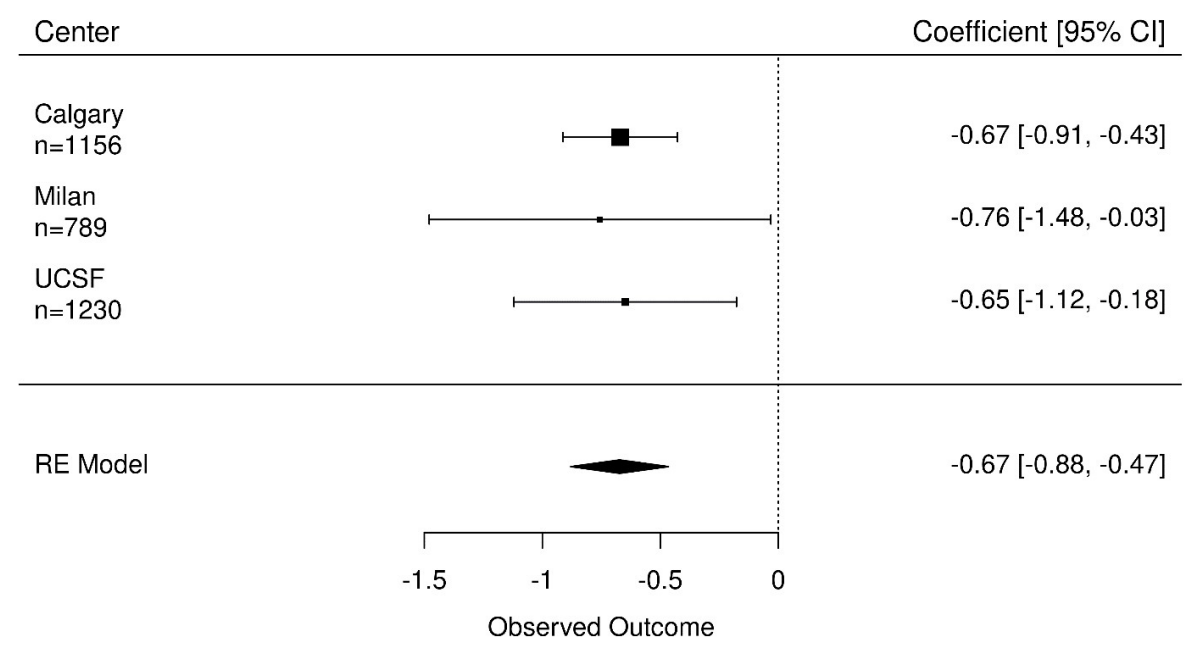

Figure 2 Coefficients of the number of biopsies on erectile functioning (SHIM/IIEF-5), n represent number of questionnaires completed. The effect of the number of biopsies on erectile functioning is controlled for age at diagnosis, time on AS and the Gleason score at diagnosis. AS, active surveillance.

known side-effects such as urinary incontinence, erectile dysfunction and potential bowel problems, patients must be aware that choosing AS may, on the long-term, not be harm-free either. Although the effects may be small, patients and urologists may anticipate a decline in erectile function with increasing numbers of follow-up biopsies. They should take that into account when expressing their preferences and subsequent treatment decision making at diagnosis, but may also use this information when deciding on the intensity of the follow-up biopsy schedule after being on AS for longer times (e.g., more than 5 years).

One of the limitations of this study is that only $3-4 \%$ of the GAP3 population was included in the current analysis and that the participating centers that are included show heterogeneity of their AS protocols. As a result, patients underwent different follow-up prostate biopsy protocols, meaning that at the University of California, San Francisco (UCSF) yearly prostate biopsies may have been taken, while 
the prostate biopsy interval at the Instituti Tumori in Milan may have been longer as they connect to the PRIAS AS protocol. However, this is likely to reflect the "real world" variance in clinical practice that is seen worldwide with respect to AS (26). Furthermore, as indicated in the results section, although different inclusion criteria and follow-up schedules were followed, the results were consistent across cohorts. A second limitation is that the GAP3 database does not specify whether biopsies were taken transrectal or transperineal. While the transrectal route is currently still the most common technique in most countries, the transperineal biopsy route is used increasingly (27). This may impact the biopsy-related complications experienced by men and hence their self-reported urinary and erectile function. Mostly single-center studies have published their results on urinary and erectile function after transperineal biopsies were taken. Most report that transperineal biopsy does not have a significant effect on patient-reported urinary and erectile function up to six months after the prostate biopsy procedure was conducted (28-34). In the studies that did saw an effect, mainly on erectile function, the effect was, however, rather small, questioning whether it is clinically relevant. With longer follow-up on an AS management strategy, and hence undergoing more prostate biopsies, it could still be that many small effects add up to a larger effect. Therefore, further research with long-term follow-up is still warranted.

In conclusion, repeated prostate biopsy as part of an AS protocol for men with low-risk PCa does not have a significant effect on self-reported UF but does impact self-reported sexual function. Further research is needed to understand whether the effect on sexual function is meaningful from a patient's perspective, but clinicians and patients should anticipate a potential decline in erectile function and hence consider incorporating the risk of this harm into their discussion about AS as a viable treatment option and also when deciding on the stringency of followup biopsy schedules with long-term AS.

\section{Acknowledgments}

Funding: This work was supported by the Movember Foundation. The funder did not play any role in the study design, collection, analysis or interpretation of data, or in the drafting of this paper.

\section{Footnote}

Provenance and Peer Review: This article was commissioned by the editorial office, Translational Andrology and Urology for the series "Expectant Management in Genitourinary Malignancies (Prostate, Bladder, Kidney)". The article has undergone external peer review.

Reporting Checklist: The authors have completed the STROBE reporting checklist. Available at http://dx.doi. org/10.21037/tau-20-1255

Data Sharing Statement: Available at http://dx.doi. org/10.21037/tau-20-1255

Conflicts of Interest: The authors have completed the ICMJE uniform disclosure form (available at http:// dx.doi.org/10.21037/tau-20-1255). The series "Expectant Management in Genitourinary Malignancies (Prostate, Bladder, Kidney)" was commissioned by the editorial office without any funding or sponsorship. Dr. MVH served as the unpaid Guest Editor of the series. Dr. MJR serves as an unpaid Associate Editor-in-Chief of Translational Andrology and Urology from Jan 2020 to Dec 2021. Dr. JH reports grants from the Movember Foundation, during the conduct of the study. The authors have no other conflicts of interest to declare.

Ethical Statement: The authors are accountable for all aspects of the work in ensuring that questions related to the accuracy or integrity of any part of the work are appropriately investigated and resolved. The study was conducted in accordance with the Declaration of Helsinki (as revised in 2013). The study was approved by institutional/ regional/national ethics/committee/ethics board of all 27 centers participating in the Movember GAP3 project. Between 2014 and 2016, the global GAP3 database was created by combining patient data from established AS cohorts worldwide. Requirements for participation included, amongst others, ethical approval for sharing digital patient data in a centralized global database and active registry of active surveillance patients. To date, 27 centers from the USA, Canada, Australasia, the UK, and Europe fulfilled the requirements for participation and joined the initiative. Each institution has obtained institution ethical approval and signed a Movember end user license agreement, an access rights principles agreement, and the commonly agreed upon GAP3 analytical plan.

Open Access Statement: This is an Open Access article distributed in accordance with the Creative Commons 
Attribution-NonCommercial-NoDerivs 4.0 International License (CC BY-NC-ND 4.0), which permits the noncommercial replication and distribution of the article with the strict proviso that no changes or edits are made and the original work is properly cited (including links to both the formal publication through the relevant DOI and the license). See: https://creativecommons.org/licenses/by-nc-nd/4.0/.

\section{References}

1. Mottet N, van den Bergh RCN, Briers E, et al. EAUEANM-ESTRO-ESUR-SIOG Guidelines on Prostate Cancer-2020 Update. Part 1: Screening, Diagnoses, and Local Treatment with Curative Intent. Eur Urol 2021;79:243-62.

2. Sanda MG, Cadeddu JA, Kirkby E, et al. Clinically localized prostate cancer: AUA/ASTRO/SUO Guideline. Part I: Risk Stratification, Shared Decision Making, and Care Options. J Urol 2018;199:683-90.

3. Carroll PH, Mohler JL. NCCN Guidelines Updates: Prostate Cancer and Prostate Cancer Early Detection. J Natl Compr Canc Netw 2018;16:620-3.

4. Bruinsma SM, Zhang L, Roobol MJ, et al. The Movember Foundation's GAP3 cohort: a profile of the largest global prostate cancer active surveillance database to date. BJU Int 2018;121:737-44.

5. Cooperberg MR, Carroll PR. Trends in management for patients with localized prostate cancer, 1990-2013. JAMA 2015;314:80-2.

6. Cooperberg MR. Active surveillance for low-risk prostate cancer - an evolving international standard of care. JAMA Oncol 2017;3:1398-9.

7. Tosoian JJ, Mamawala M, Epstein JI, et al. Active surveillance of grade group 1 prostate cancer: long-term outcomes from a large prospective cohort. Eur Urol 2020;77:675-82.

8. Klotz L, Vesprini D, Sethukavalan P, et al. Long-term follow-up of a large active surveillance cohort of patients with prostate cancer. J Clin Oncol 2015;33:272-7.

9. Loeb S, Vellekoop A, Ahmed HU, et al. Systematic review of complications of prostate biopsy. Eur Urol 2013;64:876-92.

10. Glass AS, Porten SP, Bonham M, et al. Active surveillance: does serial prostate biopsy increase histological inflammation? Prostate Cancer Prostatic Dis 2013;16:165-9.

11. Hilton JF, Blaschko SD, Whitson JM, et al. The impact of serial prostate biopsies on sexual function in men on active surveillance for prostate cancer. J Urol 2012;188:1252-8.

12. Burns JF, Hurwitz LM, Levie KE, et al. Impact of subsequent biopsies on comprehensive health related quality of life in patients with and without prostate cancer. J Urol 2019;201:916-22.

13. Zisman A, Leibovici D, Kleinmann J, et al. The impact of prostate biopsy on patient well-being: a prospective study of pain, anxiety and erectile dysfunction. J Urol 2001;165:445-54.

14. Fujita K, Landis $\mathrm{P}, \mathrm{McNeil} \mathrm{BK}$, et al. Serial prostate biopsies are associated with an increased risk of erectile dysfunction in men with prostate cancer on active surveillance. J Urol 2009;182:2664-9.

15. Braun K, Ahallal Y, Sjoberg DD, et al. Effect of repeated prostate biopsies on erectile function in men on active surveillance for prostate cancer. J Urol 2014;191:744-9.

16. Pearce SM, Wang CH, Victorson DE, et al. A longitudinal study of predictors of sexual dysfunction in men on active surveillance for prostate cancer. Sex Med 2015;3:156-64.

17. Barry MJ, Fowler FJ Jr, O'Leary MP, et al. The American Urological Association symptom index for benign prostatic hyperplasia. The Measurement Committee of the American Urological Association. J Urol 1992;148:1549-57.

18. Barry MJ, Williford WO, Chang Y, et al. Benign prostatic hyperplasia specific health status measures in clinical research: how much change in the American Urological Association symptom index and the benign prostatic hyperplasia impact index is perceptible to patients? J Urol 1995;154:1770-4.

19. Badía X, Garcia-Losa M, Dal-Re R. Ten-language translation and harmonization of the International Prostate Symptom Score: developing a methodology for multinational clinical trials. Eur Urol 1997;31:129-40.

20. Rosen RC, Cappelleri JC, Smith MD, et al. Development and evaluation of an abridged, 5 -item version of the International Index of Erectile Function (IIEF-5) as a diagnostic tool for erectile dysfunction. Int J Impot Res 1999;11:319-26.

21. R Core Team. R: a language and environment for statistical computing. 3.6.0. ed. Vienna, Austria: R Foundation for Statistical Computing; 2018.

22. Viechtbauer $\mathrm{W}$. Conducting meta-analyses in $\mathrm{R}$ with the Metafor Package. Journal of Statistical Software 2010;36:48.

23. Erler NS, Rizopoulos D, Jaddoe VWV, et al. Bayesian imputation of time-varying covariates in linear mixed models. Statistical Methods in Medical Research 2019;28:555-68.

24. Mottet N, van den Bergh RCN, Briers E, et al. EAU Guidelines: Prostate Cancer. Available online: https:// 
uroweb.org/guideline/prostate-cancer. Accessed on March 30, 2020.

25. Barry MJ, Edgman-Levitan S. Shared decision makingpinnacle of patient-centered care. N Engl J Med 2012;366:780-1.

26. Bruinsma SM, Bangma CH, Carroll PR, et al. Active surveillance for prostate cancer: a narrative review of clinical guidelines. Nat Rev Urol 2016;13:151-67.

27. National Prostate Cancer Audit. Sixth Year Annual Report 2019. Available online: https://www.npca.org.uk/content/ uploads/2020/01/NPCA-Annual-Report-2019_090120.pdf

28. García Rojo E, Garcia Gomez B, Gonzalez Padilla DA, et al. Assessment of the influence of transrecta land transperineal prostate biopsies on erectile function: a prospective observational single-center study. Int J Urol 2019;26:1054-8.

29. Dowrick AS, Wootten AS, Howard N, et al. A prospective study of short-term quality of life outcomes of patients undergoing transperineal prostate biopsy. BJU Int

Cite this article as: Crump RT, Remmers S, Van Hemelrijck M, Helleman J, Nieboer D, Roobol MJ, Venderbos LDF; Movember Foundation's Global Action Plan Prostate Cancer Active Surveillance (GAP3) consortium. Using the Movember Foundation's GAP3 cohort to measure the effect of active surveillance on patient-reported urinary and sexual functiona retrospective study in low-risk prostate cancer patients. Transl Androl Urol 2021;10(6):2719-2727. doi: 10.21037/tau-20-1255
2016;118:60-7.

30. Losa A, Gadda GM, Lazzeri M, et al. Complications and quality of life after template-assisted transperineal prostate biopsy in patients eligible for focal therapy. Urology 2013;81:1291-6.

31. Merrick GS, Irvin S, Fiano R, et al. Pathology and quality of life outcomes following office-based transperineal prostate biopsy. Urology 2016;94:24-8.

32. Miah S, Eldred-Evans D, Simmons LAM, et al. Patient reported outcome measures for transperineal template prostate mapping biopsies in the PICTURE study. J Urol 2018;200:1235-40.

33. Pepe P, Pennisi M. Erectile dysfunction in 1050 men following extended (18 cores) vs saturation (28 cores) vs saturation plus MRI-targeted prostate biopsy (32 cores). Int J Impot Res 2016;28:1-3.

34. Tsivian M, Abern MR, Qi P, et al. Short-term functional outcomes and complications associated with transperineal template prostate mapping biopsy. Urology 2013;82:166-70. 